\title{
Automatisierungssysteme für das digitale Zeitalter
}

\begin{abstract}
Die weltweiten Datenmengen wachsen im Zuge der Digitalisierung exponentiell. Immer mehr industrielle Prozesse werden mit Sensoren überwacht, analysiert und automatisiert. Mit einer neuen Cloud und der passenden Softwarelösung begleitet Jumo Unternehmen auf dem Weg zur intelligenten Fabrik der Zukunft.
\end{abstract}

Der Hersteller für Mess-, Regel und Automatisierungstechnik aus Fulda entwickelt sich bereits seit Jahren zu einem Anbieter von branchenspezifischen Komplettlösungen und produziert hierfür auch die komplette Hardware. Das beginnt mit Sensoren für verschiedene physikalische Größen und führt über Regler und Bildschirmschreiber bis hin zu leistungsfähigen Automatisierungssystemen. Die Cloud ist der nächste logische Schritt in dieser Entwicklung. Sie basiert auf dem Modell, Software as a Servic' (SaaS) und ist eine cloudbasierte Anwendungssoftware mit einer vordefinierten Benutzeroberfläche. Als IoT-Plattform zur Prozessvisualisierung, Datenerfassung, -auswertung sowie -archivierung ermöglicht die Cloud den weltweiten Zugriff auf Messdaten über die gängigen Webbrowser. Sie zeichnet sich durch hohe Sicherheit und wertvolle Visualisierungs-, Alarm- und Planungsfunktionen aus. Kunden können mit Hilfe der Cloud mehrere verteilte Anlagen, Prozesse oder Standorte in einem Dashboard überwachen und so die Prozesssicherheit erhöhen.

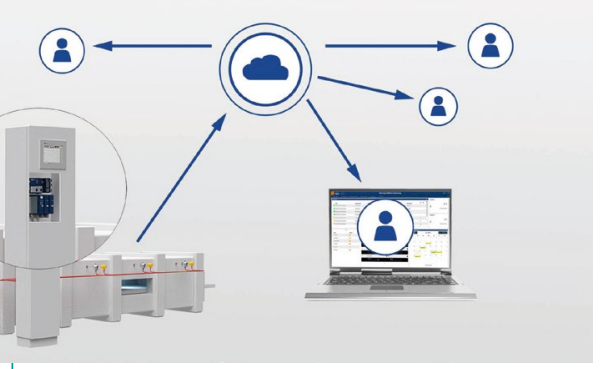

Mit der Cloud und dazugehöriger Softwarelösung eröffnet Jumo neue Automatisierungsmöglichkeiten. (ㄷ) Jumo)

\section{Komplette Anlagensteuerung} aus der Cloud

Da Jumo die Datenspeicherung mit einer redundanten und zertifizierten Infrastruktur übernimmt, ergibt sich für Anwender auch eine signifikanteZeitersparnis. Durch professionelle Report- und Exportfunktionen kann der Aufwand für nachweispflichtige Datenerfassungen deutlich reduziert werden. Alle DSGVO-Standards werden durch die ausschließliche Zusammenarbeit mit europäischen Rechenzentren erfüllt. Die Möglichkeiten der Cloud reichen von einfachen Alarmmeldungen über ein Condition-Monitoring bis hin zu kompletten Anlagensteuerungen. Die Cloud ist dabei auf die neue Hard- und Softwareplattform Jupiter von Jumo zugeschnitten, die das Kernstück des ebenfalls neuen Automatisierungssystems varitron ist.

\section{Plattform visualisiert Prozesse}

Parallel zur Cloud-Lösung wurde die smartware Scada entwickelt. Diese Softwarelösung auf Basis der Cloud ist in der Automatisierungspyramide auf der Leitebene angesiedelt. Scada ermöglicht Zugriff auf Messdaten über gängige Webbrowser und bietet Funktionen zur Prozessvisualisierung sowie zur Auswertung und Archivierung der erfassten Daten. Die Visualisierung erfolgt mit Hilfe eines Editors mit integriertem Animationsund Test-Tool sowie vektorbasierten, selbstskalierenden Prozessbildern.

Scada ist somit eine hochskalierbare und performante Digitalisierungs-Plattform, die Herstellungs- und Arbeitsprozesse mit effizienten Visualisierungs-, Alarm- und Planungsfunktionen unterstützt. Aufgrund einer modernen Web-Oberfläche ist ein Cli-

\section{Über Jumo}

Jumo mit Hauptsitz in Fulda ist ein HighTech-Anbieter von Produkten im Bereich der Mess-, Regel und Automatisierungstechnik. Als familiengeführtes Unternehmen in der dritten Generation beschäftigt das Unternehmen heute weltweit über 2.400 Mitarbeiter. Das Produktspektrum umfasst die gesamte Messkette vom Sensor bis zur Automatisierungslösung für die Messgrößen Temperatur, Druck, Flüssigkeitsanalyse, Durchfluss, Füllstand und Feuchte.

ent-Zugriff ohne Installation möglich. Als mandantenfähiges System verfügt sie darüber hinaus über individuell einstellbare Nutzerrechte. Die Sicherheit ist durch eine durchgängige Verschlüsselung mit möglicher Zwei-Faktor-Authentifizierung nach aktuellem Stand der Technik gewährleistet.

Bei der Nutzung der Cloud kann der Kunde zwischen einer Flatrate und einem Pay-per-Use-Modell entscheiden. Der Cloud-Speicher lässt sich hierbei flexibel für die jeweiligen Anforderungen erweitern und anpassen. Auch bei der Softwarelösung sind variable und individuelle Preisgestaltungen möglich. Die Realisierung der branchenund projektspezifischen Cloud- und Software-Applikationen bei Anwendern aus vielfältigen Branchen unterstützt das Engineering-Team von Jumo.

Kontakt:

JUMO GmbH, 36039 Fulda www.jumo.de 